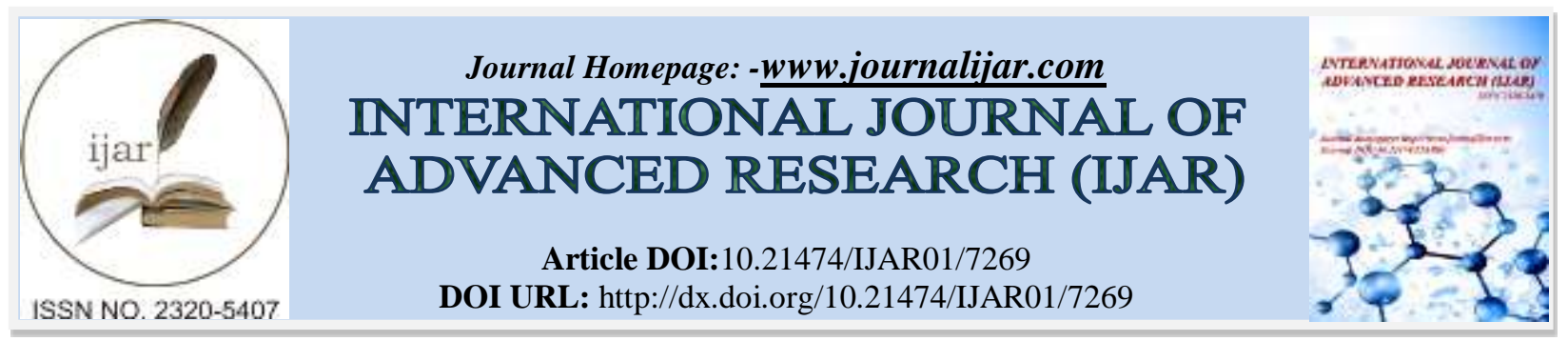

RESEARCH ARTICLE

\title{
CHONDROMA MAXILLA: A CASE REPORT.
}

\section{Veten Mohamed ${ }^{1}$, Elghazaly ma $^{2}$ and Tichitty ahmed ${ }^{1}$.}

1. Service de stomatologie et de chirurgie faciale du centre hospitalier national de Nouakchott(Mauritanie).

2. Service d'imagerie médicale du centre national des spécialités médicales.

\section{Manuscript Info}

Manuscript History

Received: 12 April 2018

Final Accepted: 14 May 2018

Published: June 2018

Keywords:-

Chondroma, Maxillary, Resection

\section{Abstract}

The chondroma is a benign cartilage tumor that rarely develops in the bones of the face. It preferentially affects the age group between 50-60 years. Its seat is mostly preferred metaphyses of long bones. The histological characteristics have enabled the diagnosis of chondroma of the jaw for this tumor. The treatment of the lesion consisted of a complete surgical excision and postoperative course was simple.

Copy Right, IJAR, 2018,. All rights reserved.

\section{Introduction:-}

Le chondrome est une tumeur bénigne cartilagineuse qui se développe rarement au niveau des os de la face, elle touche préférentiellement, les métaphyses des os tubulaires, L'histogénèse de cette tumeur est encore discutée, étant donné la nature membraneuse des structures osseuses maxillaires.

Le traitement de cette tumeur consiste en une exérèse chirurgicale complète.

Nous rapportons un cas d'un chondrome géant du maxillaire, déformant l'hémiface et entrainant des chutes et des déplacements dentaires.

\section{Observation:-}

Il s'agit d'une patiente, âgée de 58 ans sans passé médical particulier, d'un niveau socio-économique modeste, venue consultée pour une tuméfaction maxillaire, évoluant, depuis 2 ans, indolore, d'évolution lente et de consistance dure, déformant l'hémiface gauche, comblant tout le vestibule antérieur, n'entrainant pas de troubles sensitivomoteurs dans le territoire concerné. L'examen clinique exobuccal montre une asymétrie faciale due à une tuméfaction du maxillaire antérieur plus marquée à gauche, qui soulève la lèvre supérieure et efface le sillon nasogénien gauche.

A l'examen endobuccal, on note une tumeur bourgeonnante, recouverte d'une muqueuse érythémateuse par endroit. Les aires ganglionnaires cervicaux sont libres.

L'examen tomodensitométrique a révélé l'existence d'une masse jouxta corticale de l'os maxillaire massivement calcifiée avec des calcifications ponctuées faisant évoquer une nature cartilagineuse sans envahissement du sinus maxillaire.

Le traitement a consisté en une exérèse chirurgicale complète de la tumeur et les suites opératoires ont été simples. Ce traitement a permis le contrôle local de la tumeur sans récidive après 5 ans. 


\section{Discussion:-}

Le chondrome du maxillaire est une tumeur bénigne d'étiologie inconnue $(15,12)$, décrite pour la première fois par Muller en 1838, l'affection est rare au niveau du complexe maxillo-mandibulaire, les localisations préférentielles sont par ordre de fréquence décroissante, la région antérieure du maxillaire supérieur, la région symphysaire et la région angylo-mandibulaire(12), quand la lésion est Centro-osseuse on parle d'enchondrome dans le cas contraire c'est le chondrome juxta-cortical.

Plusieurs syndromes sont associés a des chondromes en particulier le syndrome d'Ollier qui est une enchondromatose multiples de localisation métaphysaire (11), rare au niveau des maxillaires et le syndrome de maffucci associant des hémangiomes aux enchondromes. (10)

Le chondrome se présente comme une tumeur indolore, d'évolution lente qui peut prendre des mois voir des années, cette tumeur est respectueuse des structures de voisinages en les refoulent sans les envahir, il peut entrainer d'importantes déformations faciales ainsi que des chutes et des mobilités dentaire associées. $(6,15)$

Radiologiquement la tumeur se manifeste par une ostéolyse diffuse, parfois inhomogène en raison de condensations irrégulières ou de fines trabéculations intralésionnelles, les réactions d'ostéocondensations sont très rares. $(8,13)$

L'imagerie par résonance magnétique reste l'imagerie de choix pour étudier cette tumeur mais pour des raison technique cet examen n'as pas pu être réaliser et ont s'est limiter a la tomodensitométrie qui a révélée l'existence d'une massejouxta corticale de l'os maxillaire massivement calcifiée avec des calcifications ponctuées faisant évoquer une nature cartilagineuse sans envahissement du sinus maxillaire(6,3).

Ces caractéristiques radiologiques orientent vers le diagnostic d'une tumeur cartilagineuse bénigne mais posent la difficulté du diagnostic différentiel avec les autres tumeurs cartilagineuses bénignes des maxillaires en particuliers les l'ostéochondrome, les chondroblastome et le fibrome chondromyxoïde ou seul l'examen anatomopathologique permet leurs diagnostic de certitude. (15) .

Histologiquement le chondrome est constitué de lobules hyalins composés de petits chondrocytes contenus chacun dans une logette, visibles au sein d'une substance chondroïde homogène ou d'un stroma myxoïde. (9) Ces cellules ne présentent qu'un pléomorphisme minime et une binucléation rare, sans atypie nucléaire associée. Quelques calcifications sont présentes de façon éparse. (9), (12)

Cette analyse histologique ne peut pas écarter formellement le diagnostic de chondrosarcome car $20 \%$ des chondrosarcomes de l'extrémité céphalique sont considérés initialement comme des chondromes. (15)

Le traitement de la lésion a consisté en une exérèse chirurgicale complète. En effet, le traitement du chondrome maxillaire est chirurgical.(12) L'exérèse doit être complète et radicale d'emblée, pour éviter les récidives redoutable en absence d'exérèse totale.

La radiothérapie et la chimiothérapie n'ont pas de place dans l'arsenal thérapeutique du chondrome du maxillaire. (11)

Les contrôles cliniques et radiologiques postopératoires ce sont étalés sur 5 ans sans notés de nouvelles récidives. 


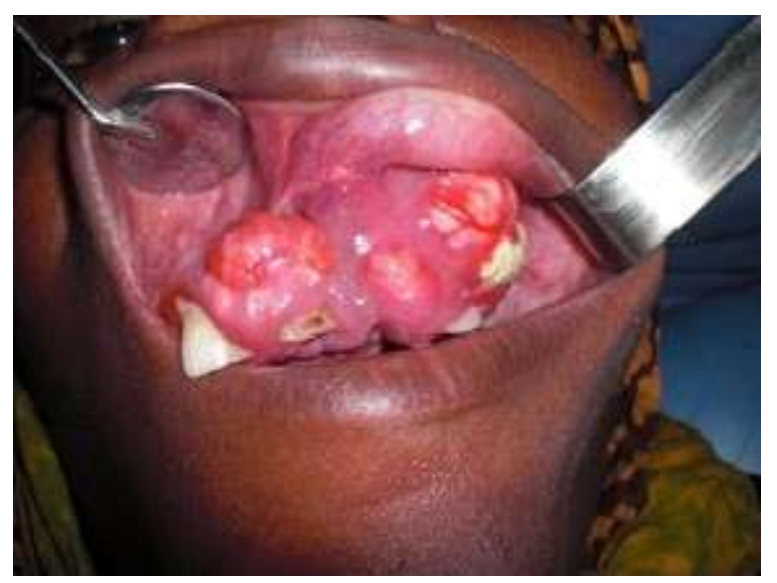

Figure 2:-Vue Endobuccale

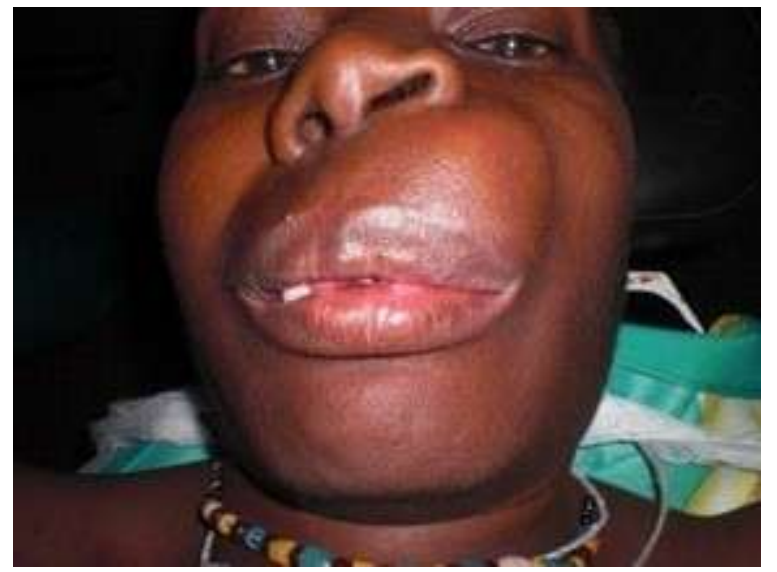

Figure 1:-vue exobuccale

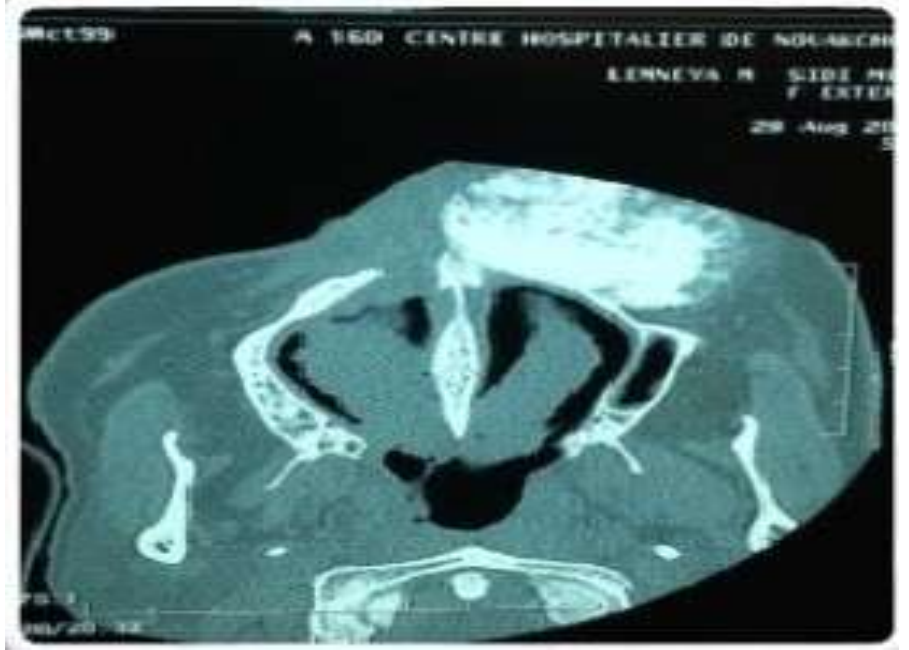

Figure 3:-TDM maxillaire en coupe axiale 


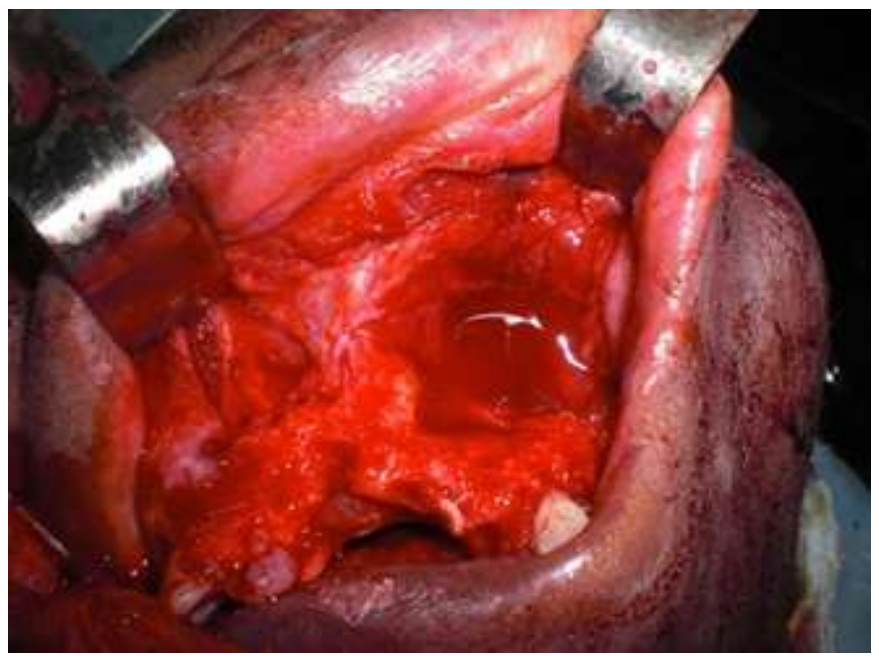

Figure 4:-vue peropératoire

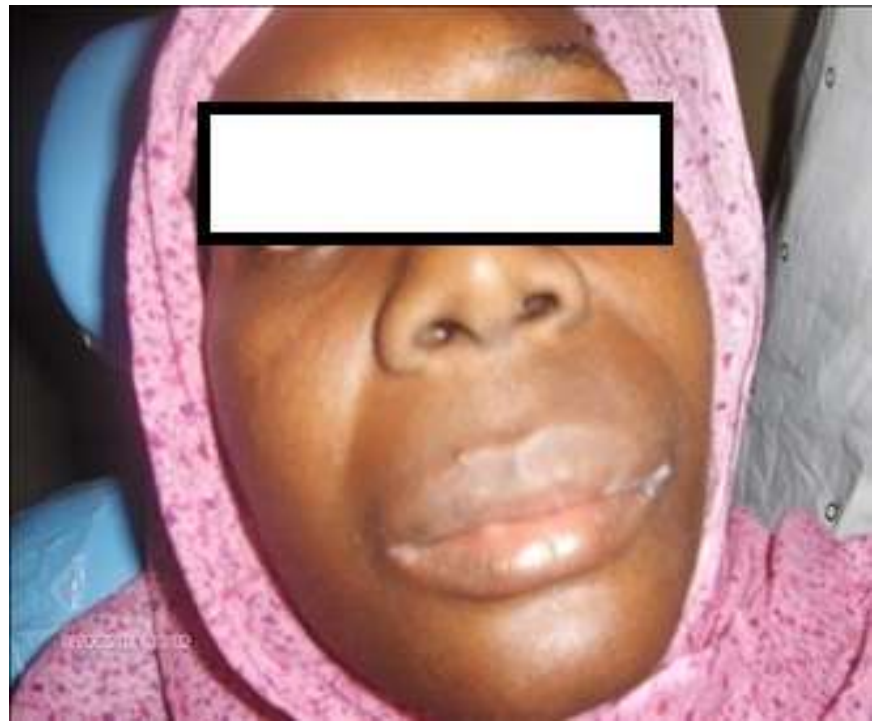

Figure 6:-contrôle a 15 jours de 1 intervention

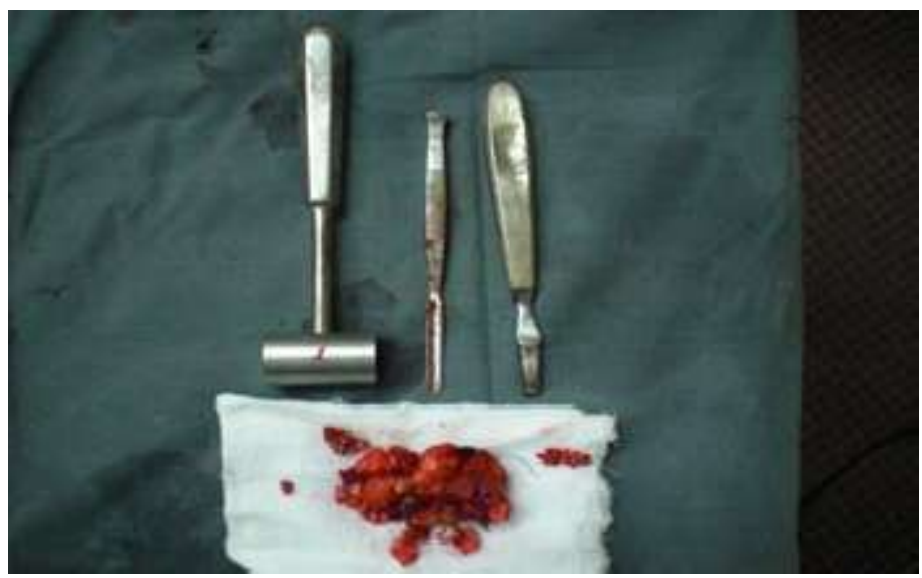

Figure 5:-pièce opératoire 


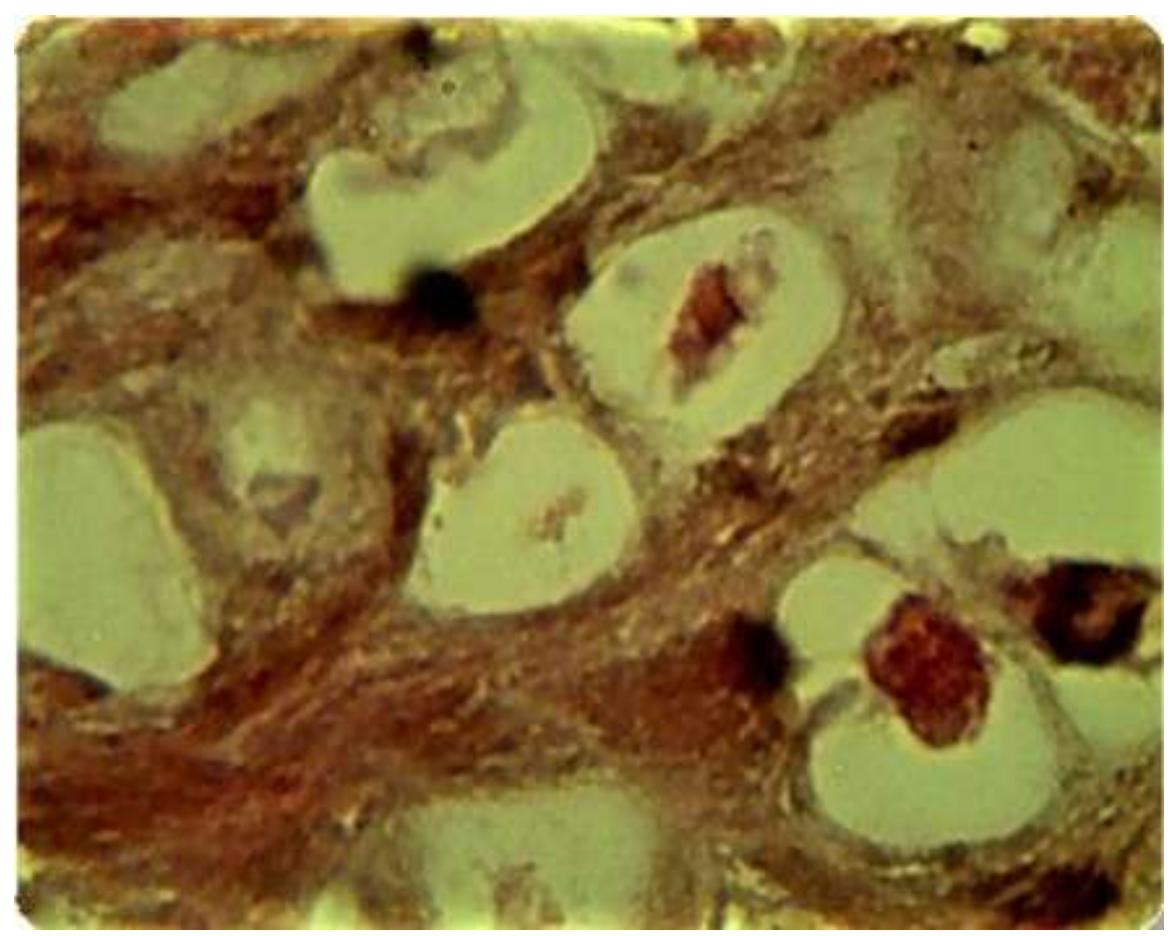

Figure 7:-chondrocyte dans leurs logettes

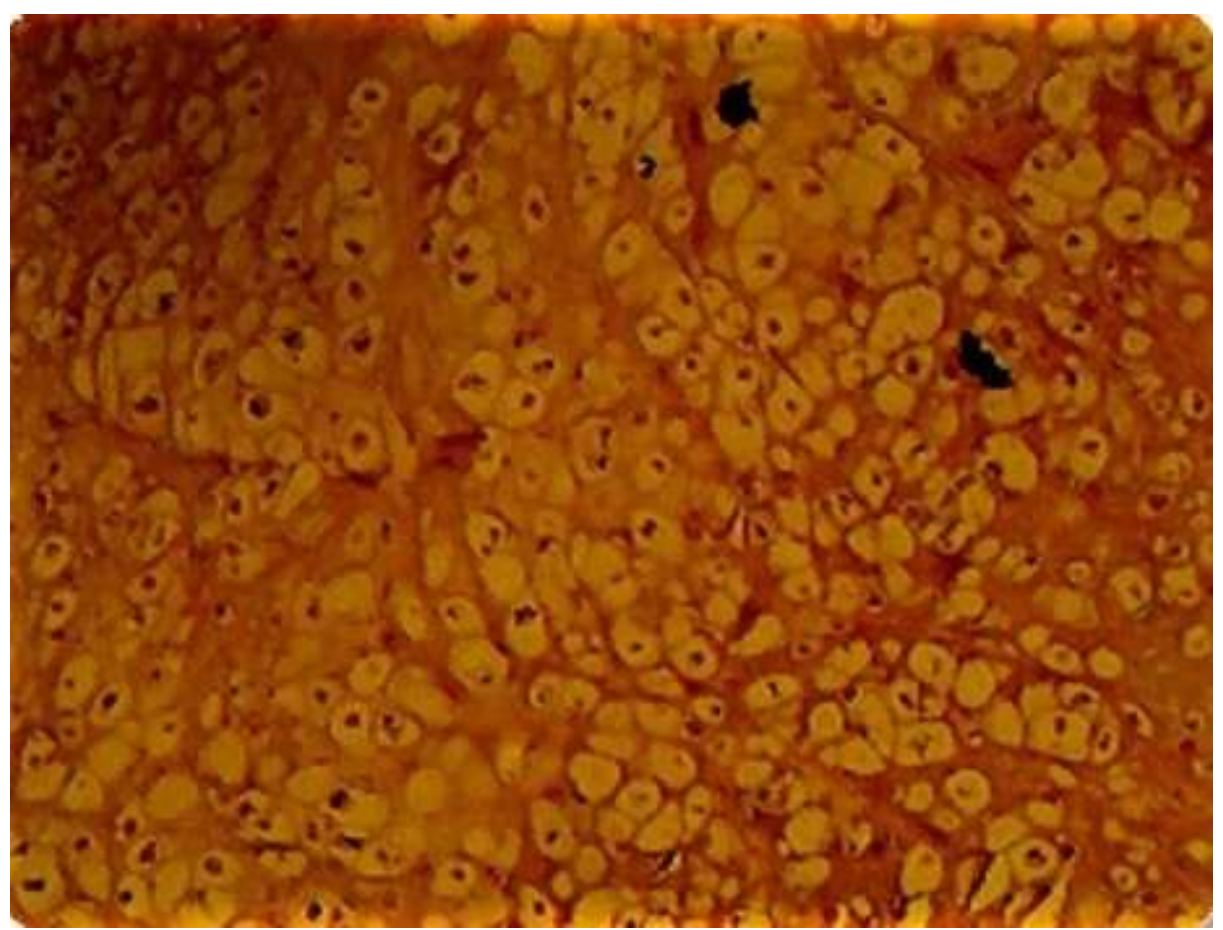

Figure 8:-Charpente Conjonctive 


\section{Bibliographie:-}

1. Aydin ma, kuçukçelebi a, sayikan s, celebiogly s. Osteochondroma of the mandiblar condyle: report of 2 cases treated with conservative surgery.j. Oral. Maxillofac. Surg. 2001; 59(9):1082-9

2. Brady fa, sapp jp, christensen re. Extracondylarosteochondromas of the jaws. Oral surg. Oral med. Oral pathol. 1978; 46(5):658-68.

3. Brygo a, leroy $x$, maes $j-m$, ferri $j$. Tumeurs et pseudotumeurs non odontogènes bénignes des maxillaires.Encycl. Médic. Chir.stomatol. 22-062-h-10, 2006.

4. Diviyalakshmi mr, iyengar ar, nagesh ks.Chondrosarcoma of the maxilla: report of a case.An. Dent. Res. 2012; 2(2):79-85.

5. Ide f. Chondromyxoid tumor of palate. J. Oral pathol. Med. 2006; 35(8):523-4.

6. J.p. Crestanellonese, c. Fernándezluzardo, c. ArismendiRev esp cir oral y maxilofac 2006;28,5 (septiembreoctubre):295-300 @ 2006 ergon

7. Kawano t, yanamoto s, kawasakia g, mizunoa a, fujita s, ikeda t. Soft tissue chondroma of the hard palate: a case report.As. J. Oral maxillofac. Surg. 2011; 23 (2):92-5.

8. Kermer ch, rasse m, undt g, lang sCartilaginous exostoses of the mandible.Int. J. Oral maxillofac. Surg. 1996; 24(3):184-

9. Martin-duverneuil $\mathrm{n}$, auriol $\mathrm{m}$. Le tumeurs maxillo-faciales : image, anatomopathologie. Montpellier : sauramps médical ; $2004 ; 402 \mathrm{p}$.

10. maffucci a. Di un casoencondromaedangiomamultiplo ;Contribuzionz alla genesiembrionale dei tumori. ovimentoMed chir1881;3:399-412.

11. Ollier m. Sur une nouvelle affection : la dyschondroplasie. RevChir paris 1900;21:396-8.

12. Piette e, reychler h. Pathologie des maxillaires. Traité de pathologie buccale et maxillofaciale. Bruxelles : de boeck université $2002 ; 1331-1436 \mathrm{p}$

13. Ramanathan k, keat tc, singh h.Chondroma of the palate. Case report. Aust dent. J. 1970; 15:478-81

14. Snyder sr, merkow lp. Benign chondroma of the palate: report of case.J. Oral surg. 1973; 31:873-5.

15. Taupin a, soubeyrand e, garmi $\mathrm{r}$, traoré $\mathrm{h}$, compère jf, bénateau h.Tumeurs cartilagineuses bénignes des mâchoires.Rev. Stomatol. Chir. Maxillofac. 2009; 110(5):290-2. 